\title{
ESTIMATION OF THE TIME OF ATTAINMENT OF A THRESHOLD WITH CONTROL OF THE PROBABILITY OF OVERESTIMATION
}

\author{
Isaac Bekele \\ Faculty of Agriculture \\ University of West Indies \\ St. Augustine, Trinidad \\ West Indies
}

Charles E. McCulloch

\author{
Biometrics Unit and Statistics Center \\ Cornell University \\ Ithaca, NY 14853 \\ U.S.A.
}




\title{
ESTIMATION OF THE TIME OF ATTAINMENT OF A THRESHOLD WITH CONTROL OF THE PROBABILITY OF OVERESTIMATION
}

\author{
Isaac Bekele \\ Charles E. McCulloch \\ Faculty of Agriculture \\ University of West Indies \\ St. Augustine, Trinidad \\ West Indies \\ Biometrics Unit and Statistics Center \\ Cornell University \\ Ithaca, NY 14853 \\ U.S.A. \\ Key words and phrases: percentile; sequential sampling; stochastic approximation; \\ stopping rule.
}

\begin{abstract}
Consider a random variable whose mean value depends on time such as the mean germination rate of seeds stored in a seed bank. Interest lies in the estimation of the time at which the mean of the random variable attains a known fixed value when overestimation is considered to be more costly than underestimation. We assume the random variable under study has a mean value that is a linear function of time with i.i.d. normal errors.

Estimates based on sequential sampling carried out at prespecified sampling times tend to be more costly than those based on estimated inspection times since frequent sampling increases sampling cost and infrequent sampling could lead to a high probability of stopping past the desired time and a large average distance past the desired time. Among three procedures based on estimated inspection times a procedure based on inspection times determined exactly after updating the information performed better than the other two in terms of a lower sample size requirement. The probability of stopping past the desired time was slightly inflated for this procedure but the average distance from the desired time of those estimates which exceeded it was very small.

The existing procedure adopted for the current formulation and setup controlled the probability of stopping late at the desired level and performed better for small values of the slope. Compared to a procedure based on specific constants selected to control the probability of stopping late at the desired level for each slope, the sample size requirement of the existing procedure was higher, ranging from $616 \%-2493 \%$ higher. This higher sample size requirement did not lead to an appreciable advantage when the slope is not small.
\end{abstract}




\section{INTRODUCTION}

Consider a random variable whose mean value depends on time. The problem of estimation of the time that the mean attains a known fixed threshold value will be considered when the cost of overestimation is high. For instance it is not desirable to maintain seeds stored in gene banks beyond the time that a proportion of seeds failing to germinate is above a certain prespecified level. It would be necessary to terminate or regulate the exploitation of a renewable resource if the remaining amount falls to a critical level. In such cases it is necessary to use an estimator that rarely overestimates and has a small average distance beyond the time at which the threshold value is attained.

The stochastic approximation rule developed by Robbins and Monro (1951) is a recursion rule for estimation of the time that the mean of the random variable of interest attains a prespecified critical value in a regression setting where over- or underestimation of the time of interest has equal cost and samples are allowed to go back in time. It is therefore not appropriate for our interest.

Wu (1986) has indicated that the small sample properties of an adaptive Robbins-Monro (RM) scheme can be improved by maximum likelihood (ML) recursion. Sellke (1986a, 1986b) has also investigated ML recursion in quantal response and examined the consistency of ML recursion. Confidence interval estimation of the parameter of interest has been considered by Ruppert (1988). The ML recursion rule, like its predecessor, the RM scheme, does not take cost in to consideration.

For the seed bank situations, repeated fixed sample size test (ISTA, 1976a, $1976 \mathrm{~b})$ and the sequential probability ratio tests (1981) have been proposed. These procedures were developed by formulating the problem as a hypothesis test. A sequential procedure based on estimated inspection times has also been proposed (Whitehead, 1989) and its properties investigated using the Monte Carlo method.

Unless inspections are carried out frequently, at least in the neighborhood of the time that the threshold is attained, procedures based on prespecified grids are likely to terminate beyond the required time more frequently than desired and the residual life could be too high. On the other hand, frequent inspection could control the frequency of terminating beyond the desired time but could lead to a high sampling cost which may be unaffordable. 
Here, estimators of the time of attainment of a given threshold value based on a sequential procedure and estimated inspection times will be given and their properties evaluated using computer simulation when the mean of the random variable of interest is assumed to be a linear function of time with a known intercept. The properties of these estimates will be compared to those of Whitehead's (1989) adapted for this situation. The inspection times computed by Whitehead's approach have the desired time of termination as their $(1-\alpha)^{t h}$ percentile for $\alpha \in(0,0.05)$ and the procedure is devised to control the probabilities of late and early termination.

\section{CONSTRUCTION OF AN ESTIMATOR OF $\mathrm{t}^{*}$}

For $t \geq 0$, let $y(t)$ denote a random variable of interest and $\mu(t)$ be its mean value at time t. For a given fixed constant $\mu^{*}$ it is assumed that there exists a constant $t^{*}$ that satisfies

$$
\mu\left(\mathrm{t}^{*}\right)=\mu^{*} \text {. }
$$

At stage $n(n \geq 1)$ a fixed sample Bayes rule for testing the hypothesis,

$$
\mathrm{H}_{0 \mathrm{n}}: \mu\left(\mathrm{t}_{\mathrm{n}}\right) \geq \mu^{*} \quad \text { vs. } \quad \mathrm{H}_{\mathrm{An}}: \mu\left(\mathrm{t}_{\mathrm{n}}\right)<\mu^{*},
$$

was derived for a prespecified inspection time $t_{n}$. A sequential procedure whose form of stopping rule was that of the fixed sample Bayes was used in the construction of an estimate of $t^{*}$ assuming that the sequence of inspection times represented by $t_{n}$ is fixed in advance. For some $\alpha \in(0,0.5)$, we wish the estimator to have $1-\alpha$ percentile equal to $\mathrm{t}^{*}$ so that the probability of overestimation is $\alpha$.

Let $\mathrm{y}_{1}, \mathrm{y}_{2}, \cdots, \mathrm{y}_{n}$ denote a random sample each drawn, respectively, at times $\mathrm{t}_{1}, \mathrm{t}_{2}, \cdots, \mathrm{t}_{n}$. It was assumed that:

(i) $E\left(\mathrm{y}_{i}\right)=\beta \mathrm{t}_{i} \quad \mathrm{i}=1(1) \mathrm{n}$

(ii) The joint conditional distribution of $\mathrm{y}_{1}, \mathrm{y}_{2}, \cdots, \mathrm{y}_{n}$ given $\beta$ is normal with a vector of means given in (2.1) and variance $I \sigma^{2}$, where $\sigma^{2}$ is assumed known.

(iii) $\beta$ has a normal prior with mean $\mu_{\beta}$ and variance $\tau^{2}$.

At stage $n$, a Bayes rule would accept the null hypothesis if

$$
\mathrm{s}_{n} \geq c \sigma \sqrt{\mathrm{v}_{n}+\frac{\sigma^{2}}{\tau^{2}}}+\frac{\mu^{*}}{\mathrm{t}_{n}} \mathrm{v}_{n}+\left(\frac{\mu^{*}}{\mathrm{t}_{n}}-\mu_{\beta}\right) \frac{\sigma^{2}}{\tau^{2}}
$$

and rejects otherwise, where $c$ is a constant that depends on the relative cost associated with the assumed constant loss function and 


$$
\mathrm{s}_{n}=\sum_{i=1}^{n} \mathrm{y}_{i} \mathrm{t}_{i} \quad \text { and } \quad \mathrm{v}_{n}=\sum_{i=1}^{n} \mathrm{t}_{i}^{2}
$$

Apart from $c$, the right-hand side of expression (2.2) contains $\mu_{\beta}$ and $\tau$ which are unknown constants. Setting $\mathrm{c}_{1}=\tau^{-2}$ and $\mathrm{c}_{2}=\left(\mu^{*} / \mathrm{t}_{n}-\mu_{\beta}\right) / \tau$ and replacing $\sigma$ by one since it is assumed known the Bayes rule would accept the null hypothesis if

$$
\mathrm{s}_{n} \geq \mathrm{c} \sqrt{\mathrm{v}_{n}+\mathrm{c}_{1}}+\frac{\mu^{*}}{t_{n}} v_{n}+c_{2} \sqrt{\mathrm{c}_{1}}
$$

and rejects otherwise. Here only the case where $c_{1}=0$, which is equivalent to assuming a noninformative prior for $\beta$ will be considered. Thus, at stage $\mathrm{n}$ the fixed sample Bayes rule accepts the null hypothesis if

$$
\mathrm{s}_{n} \geq \mathrm{c} \sqrt{\mathrm{v}_{n}}+\frac{\mu^{*}}{\mathrm{t}_{n}} \mathrm{v}_{n}
$$

and rejects otherwise.

When $\mathrm{n}=1$, a sample is drawn and monitoring is terminated by declaring $\mathrm{t}_{1}$ as an estimate of $t^{*}$ if $s_{1} \geq c \sqrt{v_{1}}+\left(\mu^{*} / t_{1}\right) v_{1}$. Otherwise monitoring continues with the second inspection at $t_{2}$. At time $t_{2}$, a sample is drawn and monitoring terminated if $s_{2} \geq c \sqrt{v_{2}}+\left(\mu^{*} / t_{2}\right) v_{2}$ and $t_{2}$ is declared an estimate of $t^{*}$. In general at stage $n$ a sample is drawn and monitoring terminated with $t_{n}$ as an estimate of $t^{*}$ if (2.3) holds. Otherwise monitoring continues at stage $(n+1)$. A stopping time corresponding to the stopping rule based on (2.3) is defined as

$$
\mathrm{K}_{\mathrm{c}} \inf \left\{\mathrm{n} \geq 1: \mathrm{s}_{n} \geq \mathrm{c} \sqrt{\mathrm{v}_{n}}+\frac{\mu^{*}}{\mathrm{t}_{n}} \mathrm{v}_{n}\right\} \text {. }
$$

The estimate of $\mathrm{t}^{*}$ based on the stopping time is $\mathrm{t}_{K_{c}}$ since termination of monitoring at stage $\mathrm{K}_{c}$ implies that the threshold has been attained.

\section{CHOICE OF c}

Since the cost of overestimation is considered to be higher, we want small $\alpha$ $[\alpha \in(0,0.5)]$, and the constant $\mathrm{c}$ should be chosen such that

$$
\mathrm{P}\left(\mathrm{t}_{K_{c}}>\mathrm{t}^{*}\right) \leq \alpha
$$

when the sequence of inspection times contains $t^{*}$. If $n\left(n=1(1) N^{*}\right)$ represents the sequence of stages where $\mathrm{t}_{N^{*}}$ denotes $\mathrm{t}^{*}$, the probability given above can be evaluated based on the distribution of $\left(s_{1}, s_{2}, \cdots, s_{N^{*}}\right)$ given $\left(t_{1}, t_{2}, \cdots, t_{N^{*}}\right)$. One can easily verify that (3.1) holds if the constant $c$ in the expression is replaced by $\Phi^{-1}(\alpha)$ where $\Phi(\mathrm{x})$ denotes the normal cdf of $\mathrm{x}$. In the sequel this value of $\mathrm{c}$ will be denoted by $\mathrm{c}_{0}$. 
At stage $n(n \geq 1)$ the procedure terminates monitoring by declaring $t_{n}$ as an estimate of $t^{*}$ if

$$
\mathrm{s}_{n} \geq \mathrm{c}_{0} \sqrt{\mathrm{v}_{n}}+\frac{\mu^{*}}{\mathrm{t}_{n}} \mathrm{v}_{n}
$$

and rejects otherwise.

The corresponding stopping time is $\mathrm{K}_{c_{0}}$ and the estimate of $\mathrm{t}^{*}$ based on $\mathrm{c}_{0}$ is given by $t_{K_{c_{0}}}$. When the sequence of inspection times includes $\mathrm{t}^{*}, \mathrm{t}^{*}$ is greater than or equal to the $(1-\alpha)^{t h}$ percentile of $t_{K_{c_{0}}}$.

\section{PROPERTIES OF $\mathrm{t}_{K_{c_{0}}}$}

When a sequence of prespecified inspection times that includes $t^{*}$ is given by

$$
\mathrm{t}_{\mathrm{i}}=\mathrm{i}, \quad \mathrm{i}=1,2, \ldots,
$$

then there exists an integer $\mathrm{N}^{*}$ such that $\mathrm{t}^{*}=\mathrm{N}^{*}$ and as $\beta \rightarrow \infty$, the following hold

$$
\begin{aligned}
& \mathrm{P}\left(\mathrm{t}_{K_{c_{0}}}=\mathrm{t}_{n}\right)=\left\{\begin{array}{cl}
1-\alpha & \mathrm{n}=\mathrm{N}^{*} \\
\alpha & \mathrm{n}=\mathrm{N}^{*}+1 \\
0 & \text { otherwise }
\end{array} .\right. \\
& E\left(\mathrm{t}_{K_{c_{0}}}\right)=\mathrm{t}^{*}+\alpha \text {. } \\
& E\left(\mathrm{t}_{K_{c_{0}}}-\mathrm{t}^{*}\right)^{2}=\alpha . \\
& E\left(\left(\mathrm{t}_{K_{c_{0}}}-\mathrm{t}^{*}\right) / \mathrm{t}_{K_{c_{0}}}>\mathrm{t}^{*}\right)=0 .
\end{aligned}
$$

These results can also be established for any fixed $\beta>0$ when a sample of size $\mathrm{m}$ $(\mathrm{m}>0)$ is used at each stage as $\mathrm{m} \rightarrow \infty$ (Bekele, 1991). These properties of $\mathrm{t}_{K_{c_{0}}}$ depend on the crucial assumption that the sequence of inspection times includes $t^{*}$. In reality this assumption is unlikely to hold unless inspections are carried out continuously which is unaffordable because of high sampling cost. The effect of incorporation of estimation of inspection times on the properties of the ensuing estimator, ${ }^{t} K_{c_{0}}$ will be considered.

\section{ESTIMATION OF INSPECTION TIMES}

In this section we propose rules for estimation of inspection times which are based on the stopping rule given in (3.2). When inspection times are estimated, monitoring involves the following steps:

(i) advance specification of $t_{1}$, initial inspection time;

(ii) drawing samples; 
(iii) checking the stopping rule, if the rule points to

a. continuation of monitoring, then estimate the next inspection time using the given rule and proceed with step (ii),

b. termination, then terminate monitoring and declare the last inspection time as an estimate of $t^{*}$.

These iterations continue until monitoring is terminated by declaring the current time as an estimate of $t^{*}$. For any given stopping rule the properties of the procedures based on it depend on the rule used in the derivation of inspection times from accumulating information. Suppose at stage $n(n \geq 1)$ a decision to continue with inspection at stage $(n+1)$ has been made. Let $t$ denote a future time when monitoring will terminate. Depending on how the information to be obtained at time $t$ is treated, two approaches based on the stopping rule (3.2) are available for estimation of the next inspection time.

1. Information update approach:

Suppose at stage $n(n \geq 1)$ a decision to continue with inspection at stage $(n+1)$, at some unknown future time $t$ has been made. If monitoring is to terminate at stage $(n+1)$, at time $t$, then $y_{n+1}$ will have to be drawn and

$$
\mathrm{s}_{n+1}=\mathrm{c}_{0} \sqrt{\mathrm{v}_{n+1}}+\frac{\mu^{*}}{\mathrm{t}} \mathrm{v}_{n+1}
$$

will have to hold where $\mathrm{s}_{n+1}=\mathrm{s}_{n}+\mathrm{ty}_{n+1}$ and $\mathrm{v}_{n+1}=\mathrm{v}_{n}+\mathrm{t}^{2}$. Thus, monitoring will terminate at stage $(n+1)$ if

$$
\mathrm{s}_{n}+\mathrm{ty}_{n+1}=\mathrm{c}_{0} \sqrt{\mathrm{v}_{n}+\mathrm{t}^{2}}+\frac{\mu^{*}}{\mathrm{t}}\left(\mathrm{v}_{n}+\mathrm{t}^{2}\right)
$$

At stage $n, y_{n+1}$ is yet to be observed and will be unknown. However, since we wish to terminate monitoring at $t^{*}$, its distribution will have to be that of $\mathrm{y}_{t^{*}}$ and will have a mean $\beta \mathrm{t}^{*}$ which is $\mu^{*}$. Thus, in expression (5.1) $\mathrm{y}_{n+1}$ will be replaced by $\mu^{*}$. Replacing $\mathrm{y}_{n+1}$ and rearranging the ensuing expression, the rule that will lead to termination of monitoring at time $t$ becomes

$$
\mathrm{s}_{n}=\mathrm{c}_{0} \sqrt{\mathrm{v}_{n}+\mathrm{t}^{2}}+\frac{\mu^{*}}{\mathrm{t}} \mathrm{v}_{n} \text {. }
$$

Rule 0: For every $n \geq 1$ given $t_{1}, t_{n+1}$ is defined as

$$
\mathrm{t}_{n+1}=\inf \left\{\mathrm{t} \geq \mathrm{t}_{n}: \mathrm{s}_{n} \geq \mathrm{c}_{0} \sqrt{\mathrm{v}_{n}+\mathrm{t}^{2}}+\frac{\mu^{*}}{\mathrm{t}} \mathrm{v}_{n}\right\} \text {. }
$$

Actual computation requires a simple iteration which can be carried out quickly even by 
the least powerful computer. One can easily verify that the sequence of inspection times generated by (5.2) is monotone increasing until monitoring terminates. The sequential procedure based on the inspection times generated by (5.2) and the stopping rule (3.2) will be denoted by $\Pi_{0}$. The estimate of $t^{*}$ is $t_{K_{c_{0}}}$, where $\mathrm{k}_{c_{0}}$ is a stopping time corresponding to the stopping rule (3.2) based on a sequence of inspection times generated by (5.2).

Rule 1: The sequence of inspection times generated by (5.2) is an exact solution to

$$
\mathrm{s}_{n}=\mathrm{c}_{0} \sqrt{\mathrm{v}_{n}+\mathrm{t}^{2}}+\frac{\mu^{*}}{\mathrm{t}} \mathrm{v}_{n} \text {. }
$$

Here, given $t_{1}$, at stage $n(n \geq 1), t_{n+1}$ is defined as an approximation based on a first-order Taylor series expansion of the right-hand side of (5.3) about $\mathrm{t}=\mathrm{t}_{n}$. Thus,

$$
\mathrm{t}_{n+1}=\mathrm{t}_{n}-\left\{\frac{\widehat{\beta}_{n}-\mu^{*} / \mathrm{t}_{n}-\frac{\mathrm{c}_{0}}{\mathrm{v}_{n}} \sqrt{\mathrm{v}_{n}+\mathrm{t}_{n}^{2}}}{\mu^{*} / \mathrm{t}_{n}^{2}-\frac{\mathrm{c}_{0} \mathrm{t}_{n}}{\mathrm{v}_{n} \sqrt{\mathrm{v}_{n}+\mathrm{t}_{n}^{2}}}}\right\}
$$

where $\hat{\beta}_{n}$ is the maximum likelihood estimator of $\beta$ obtained at stage n.

The sequence of inspection times generated by (5.4) will be monotone increasing. The procedure which is based on the stopping rule given in (3.2) and the sequence of inspection times given by (5.4) will be denoted by $\Pi_{1}$. The time at which monitoring is terminated is taken as an estimate of $t^{*}$.

2. Future information exclusion approach:

Suppose at stage $n(n \geq 1)$ a decision to continue with inspection at stage $(n+1)$ has been made based on the rule (3.2). Here we seek for a future time $t$ when monitoring will terminate based only on the current information.

Rule 2: At stage $n(n \geq 1), t_{n+1}$, inspection time at stage $(n+1)$ which will lead to termination of monitoring based only on information accumulated by stage $n$ satisfies

$$
t_{n+1}=\frac{\mu^{*}}{\widehat{\beta}_{n}-\frac{c_{0}}{\sqrt{v_{n}}}} .
$$


The procedure based on inspection times generated by (5.5) and the stopping rule based on inspection times generated by (5.5) and (3.2) will be denoted by $\Pi_{2}$. At each stage, the next inspection time is defined as the lower limit of a $(1-2 \alpha) 100 \%$ confidence interval for $t^{*}$. The estimate of $t^{*}$ is the time that leads to termination of monitoring.

\section{SIMULATION RESULTS}

Properties of estimators of $t^{*}$ based on inspection times obtained using the three rules given in the preceding section were investigated using computer simulation. Also an estimator of $t^{*}$ based on Whitehead's (1989) approach derived under the current formulation and assumed distribution was included and its properties evaluated using simulation. In this case, however, corrections for overshoot were not included and the stopping boundaries which had an error in the original article were corrected. A triangular rule based on $\mathrm{Z}$ and $\mathrm{V}$ statistics defined in his equations (2.4) and (2.5), which satisfy the given requirements, would have a region of continuation given by

$$
(-8.9473+0.386 \mathrm{~V}, 8.9473+0.1287 \mathrm{~V}) \text {. }
$$

The procedure based on the continuation region given above and inspection times generated by Whitehead's approach will be denoted by $\Pi_{w}$. The following quantities were evaluated from the simulation.

(i) Probability of stopping past $\mathrm{t}^{*},(\mathrm{PSL})$ and its standard error,

$$
\mathrm{PSL}=\frac{1}{\mathrm{~N}} \sum_{j=1}^{N} I_{\left\{\mathrm{t}_{j}>\mathrm{t}^{*}\right\}} .
$$

(ii) Average sample number (ASN) and its standard error,

$$
\operatorname{ASN}=\frac{1}{\mathrm{~N}} \sum_{j=1}^{N} \mathrm{M}_{j} .
$$

(iii) Average time of termination (ATT) and its root mean square error

$$
\mathrm{ATT}=\frac{1}{\mathrm{~N}} \sum_{j=1}^{N} \mathrm{t}_{M_{j}}
$$

(iv) The mean residual life for those estimates which exceed $t^{*}$ (MRL) and its root mean square error,

$$
\mathrm{MRL}=\frac{1}{\mathrm{~N}} \sum_{j=1}^{N}\left(\mathrm{t}_{M_{j}}-\mathrm{t}^{*}\right) I_{\left\{\mathrm{t}_{j}>\mathrm{t}^{*}\right\}} .
$$

$\mathrm{N}$ represents the total number of runs and the root mean square errors were defined from $t^{*}$. 
The simulation study involved evaluation of quantities of interest for various values of $\beta$ at a fixed value of $\mathrm{t}^{*}$. The results for other values of $\beta$ and $\mathrm{t}^{*}$ can be derived from available results so long as the product of the later pair is the same as that of $\beta t^{*}$. Also simulation results results of $\Pi_{1}$ and $\Pi_{2}$ are not given here since their overall performance was poorer than that of $\Pi_{0}$.

Simulation results of $\Pi_{0}$ are given in Table 1 . In general the procedure fails to control the error rate at the desired level for values of $\beta$ that are of practical importance. Even though the error rate was slightly inflated the mean residual life of the estimate was close to zero and the average values of the estimates in most cases coincided with the parameter. The standard error associated with the average values of the terminal times decreased with increasing $\beta$.

\section{$\underline{\text { Table } 1}$}

PSL and ASN with their respective standard errors (in italics), ATT and MRL with their respective root mean square errors (in italics) obtained from 2500 simulation runs for various values of $\beta$ for $\Pi_{0}$ when $t^{*}=30$ and $t_{1}=1$.

$\begin{array}{clccl}\beta & \text { PSL } & \text { ASN } & \text { ATT } & \text { MRL } \\ 0.05 & 0.01 & 4.0 & 3.9 & 7.10 \\ & 0.002 & 0.10 & 26.8 & 8.56 \\ 0.10 & 0.07 & 12.9 & 19.6 & 2.40 \\ & 0.005 & 0.12 & 13.46 & 3.47 \\ 0.50 & 0.08 & 10.5 & 28.8 & 0.50 \\ & 0.005 & 0.07 & 1.54 & 0.61 \\ 1.50 & 0.09 & 9.4 & 29.6 & 0.10 \\ & 0.006 & 0.0 & 0.50 & 0.20 \\ 5.00 & 0.09 & 8.6 & 29.9 & 0.04 \\ & 0.006 & 0.06 & 0.15 & 0.07 \\ 10.00 & 0.09 & 8.4 & 29.9 & 0.02 \\ & 0.006 & 0.06 & 0.08 & 0.03\end{array}$

The histograms of terminal times obtained for various values of $\beta$ showed essentially three patterns. The histogram corresponding to $\beta=0.05$ showed an exponential pattern with $t_{1}=1$ as its mode. When $\beta=0.10$, a bimodal frequency distribution was observed. The rest had a typical bell shaped histogram with the center increasing to $t^{*}=30$ and a tighter spread as $\beta$ increased. 
To fairly compare $\Pi_{0}$ and $\Pi_{w}$ we must equalize PSL. For any given $t^{*}$, the constant that ensures equality of (3.1) depends on $\beta$. For values of $\beta$ used in the simulation study constants which ensured equality in expression (3.1) were searched sequentially using simulation. Procedure based on these constants denoted by $\mathrm{c}(\beta)$ and estimated inspection time generated by Rule 0 will be represented by $\Pi_{0 c(\beta)}$. Simulation results of $\Pi_{0 c(\beta)}$ and $\Pi_{w}$ are given in Table 2.

Table 2

PSL and ASN with their respective standard errors (in italics), ATT and MRL with their respective root mean square errors (in italics) obtained for various values of $\beta$ when $t^{*}=30$ and $t_{1}=1$ for $\Pi_{O c(\beta)}$ and $\Pi_{w}$ where $\Pi_{w}$ is based on 2500 runs.

\begin{tabular}{clllcccccc} 
& \multicolumn{2}{c}{ PSL } & \multicolumn{2}{c}{ ASN } & \multicolumn{3}{c}{ ATT } & \multicolumn{2}{c}{ MRL } \\
$\beta \quad$ & $\Pi_{0 c(\beta)}(\beta)$ & $\Pi_{w}$ & $\Pi_{0 c(\beta)}$ & $\Pi_{w}$ & $\Pi_{0 c(\beta)}$ & $\Pi_{w}$ & $\Pi_{0 c(\beta)}$ & $\Pi_{w}$ \\
& & & & & & & & \\
$0.05-1.155^{1}$ & 0.05 & 0.05 & 5.3 & 132.1 & 7.7 & 25.4 & 6.40 & 1.30 \\
& 0.002 & 0.004 & 0.05 & 0.40 & 24.4 & 5.35 & 8.04 & 1.75 \\
$0.10-1.730^{1}$ & 0.05 & 0.04 & 13.0 & 100.9 & 19.0 & 27.7 & 2.40 & 0.70 \\
& 0.002 & 0.004 & 0.06 & 0.24 & 14.09 & 2.66 & 3.26 & 0.98 \\
& 0.05 & 0.05 & 11.6 & 71.4 & 28.5 & 29.6 & 0.30 & 0.10 \\
& 0.003 & 0.005 & 0.05 & 0.17 & 1.70 & 0.52 & 0.50 & 0.18 \\
$1.50-1.928^{2}$ & 0.05 & 0.05 & 10.3 & 65.1 & 29.5 & 29.9 & 0.12 & 0.10 \\
& 0.003 & 0.004 & 0.05 & 0.17 & 0.56 & 0.17 & 0.17 & 0.06 \\
$5.00-1.965^{2}$ & 0.05 & 0.05 & 9.6 & 62.7 & 29.9 & 30.0 & 0.04 & 0.01 \\
& 0.003 & 0.004 & 0.05 & 0.17 & 0.17 & 0.05 & 0.05 & 0.02 \\
& 0.05 & 0.05 & 9.3 & 61.7 & 29.9 & 30.0 & 0.02 & 0.01 \\
$10.00-1.965^{2}$ & 0.003 & 0.004 & 0.05 & 0.16 & 0.08 & 0.03 & 0.03 & 0.01
\end{tabular}

${ }^{1}$ Simulation based on 10000 runs.

${ }^{2}$ Simulation based on 5000 runs.

In general, $\Pi_{w}$ controls the probability of stopping late. However, this is achieved at high cost of sampling and its average sample number ranged from 132 to 62 . The average sample number of $\Pi_{0 c(\beta)}$ ranged from 5 to 13 . The savings in sample size due to using $\Pi_{0 c(\beta)}$ ranges from $560 \%$ to $2392 \%$. The increased sample size required by $\Pi_{w}$ translates to some advantage in terms of terminating closer to the desired time and 
smaller mean residual life when $\beta$ is small. When sampling cost is high and/or test material is limited, $\Pi_{w}$ can be a costly procedure to use.

\section{CONCLUSION}

In general, the properties of the estimators based on procedures which incorporate estimation of inspection times depend on $\beta$ and $\mu^{*}$. For fixed $t^{*}$ the properties tend to improve with increasing $\beta$. $\Pi_{0}$ controls the sample size always but the associated probability of stopping late tends to be elevated slightly. However, this does not translate to increased MRL. $\Pi_{w}$ controls the probability of stopping late at the desired level but at extremely high sampling cost. For applications where sampling costs are high, as in the seed bank case $\Pi_{w}$, it will be too costly. Even in the best case its sample size requirement is six times more than that of $\Pi_{0 c(\beta)}$.

The approach pursued here can be adopted to applications with any type of quantitative factor to determine its level where a threshold is attained when cost consideration is relevant. For such cases it would be useful to investigate the asymptotic relative performance of the ensuing estimates with respect to the RM scheme. Under this formulation estimates of $t^{*}$ for cases where the variance is unknown has to be constructed.

\section{BIBLIOGRAPHY}

Bekele, I. (1991). Sequential procedures for estimation of the time of attainment of a threshold value. Ph.D Thesis. Cornell University, Ithaca, NY.

ISTA. (1976a). "International Rules for Seed Testing: Annexes 1976," Seed and Technology, 4, 3-49.

ISTA. (1976b). "International Rules for Seed Testing: Annexes 1976," Seed and Technology 4, 51-177.

Robbins, H. and Monro, S. (1951). "A stochastic approximation method," Ann. Math. Statist., 22, 400-407.

Ruppert, D. (1988). Stochastic approximation. Technical Report No. 805, School of Operations Research and Industrial Engineering, Cornell University, Ithaca, NY.

Sellke, T. (1986a). "Repeated-MLE procedures for stochastic approximation in quantal response problems," Adaptive Statistical Procedures And Related Topics, (J. Van Ryzin, Ed.). Hayward, CA: Institute of Mathematical Statistics, 283-299.

Sellke, T. (1986b). Consistency of maximum likelihood recursion in stochastic approximation. Technical Report \#86-6, Department of Statistics, Purdue University, Lafayette, IN. 
Whitehead, J. (1981). "The use of the sequential probability ratio test for monitoring the percentage germination of accessions in seed banks. Biometrics, 37, 129-132.

Whitehead, J. (1989). "Sequential methods for monitoring declining quality, with application to the long-term storage of seeds. Biometrics, 45, 13-22.

Wu, J. (1986). "Maximum likelihood recursion and stochastic approximation in sequential designs, Adaptive Statistical Procedures And Related Topics (J. Van Ryzin, Ed.). Hayward, CA: Institute of Mathematical Statistics, 298-313. 